OPEN ACCESS

Edited by:

Jagadeesh Bayry,

Institut National de la Santé et de la

Recherche Médicale (INSERM),

France

Reviewed by:

Jere W. McBride,

University of Texas Medical Branch at

Galveston, United States

Sanjay Rathod,

University of Pittsburgh, United States

${ }^{*}$ Correspondence:

Malini Sen

msen@iicb.res.in;

msen648@gmail.com

Specialty section:

This article was submitted to

Molecular Innate Immunity,

a section of the journal

Frontiers in Immunology

Received: 11 November 2020

Accepted: 21 December 2020

Published: 16 February 2021

Citation:

Jati S, Sengupta S and Sen M (2021)

Wnt5A-Mediated Actin Organization

Regulates Host Response to Bacterial

Pathogens and Non-Pathogens.

Front. Immunol. 11:628191.

doi: 10.3389/fimmu.2020.628191

\section{Wnt5A-Mediated Actin Organization Regulates Host Response to Bacterial Pathogens and Non-Pathogens}

\author{
Suborno Jati, Soham Sengupta and Malini Sen * \\ Division of Cancer Biology and Inflammatory Disorder, CSIR-Indian Institute of Chemical Biology, Kolkata, India
}

Wnt5A signaling facilitates the killing of several bacterial pathogens, but not the nonpathogen $E$. coli $\mathrm{DH} 5 \alpha$. The basis of such pathogen vs. non-pathogen distinction is unclear. Accordingly, we analyzed the influence of Wnt5A signaling on pathogenic $E$. coli $\mathrm{K} 1$ in relation to non-pathogenic E. coli $\mathrm{K} 12-\mathrm{MG} 1655$ and $E$. coli $\mathrm{DH} 5 \alpha$ eliminating interspecies variability from our study. Whereas cell internalized E. coli $\mathrm{K} 1$ disrupted cytoskeletal actin organization and multiplied during Wnt5A depletion, rWnt5A mediated activation revived cytoskeletal actin assembly facilitating $\mathrm{K} 1$ eradication. Cell internalized E. coli K12-MG1655 and E. coli DH5 $\alpha$, which did not perturb actin assembly appreciably, remained unaffected by $\mathrm{rWnt5A}$ treatment. Phagosomes prepared separately from Wnt5A conditioned medium treated K1 and K12-MG1655 infected macrophages revealed differences in the relative levels of actin and actin network promoting proteins, upholding that the Wnt5A-Actin axis operates differently for internalized pathogen and non-pathogen. Interestingly, exposure of rWnt5A treated K1 and K12-MG1655/DH5 $\alpha$ infected macrophages to actin assembly inhibitors reversed the scenario, blocking killing of K1, yet promoting killing of both K12-MG1655 and DH5 $\alpha$. Taken together, our study illustrates that the state of activation of the Wnt5A/Actin axis in the context of the incumbent bacteria is crucial for directing host response to infection.

Keywords: Wnt5A, actin, phagosome, pathogen, non-pathogen

\section{INTRODUCTION}

Wnt5A belongs to a 19-member family of Wnt ligands, which are secreted glycoproteins originally discovered with reference to embryonic development. Wnts interact with Frizzled (Fz) and ROR cell surface receptors. Frizzleds are seven transmembrane spanning receptors, about 12 in number, bearing homology to heterotrimeric $\mathrm{G}$ protein coupled receptors and RORs (ROR1 and ROR2) bear homology to tyrosine kinases (1-7). Classically, Wnt signaling is divided into two main categories canonical ( $\beta$-catenin dependent) and non-canonical ( $\beta$-catenin independent) $(6,8)$. While canonical Wnt signaling mostly acts through $\beta$-catenin mediated transactivation of specific genes, non-canonical Wnt signaling often acts independent of $\beta$-catenin during regulation of cell polarity and differentiation (9-12). On account of sequence homology within the Wnt and Frizzled/ ROR family members, cross reactivity in Wnt-Frizzled/ROR interactions and crosstalk among the signaling intermediates of the canonical and non-canonical Wnt signaling pathways is quite frequent $(7,13,14)$. 
Wnt5A, a prototype of the non-canonical Wnt signaling pathway interacts with Fz2, Fz4, Fz5 and ROR1/2 receptors regulating cell polarity and movements (2, 4, 5). Quite naturally, Wnt5A signaling is an important facet of macrophages, which respond to a broad spectrum of environmental cues including bacterial infections, through alterations in cell migration and polarity (15-17).

Macrophages are intrinsically wired to counter bacterial infections through phagocytosis, which utilizes the coordination of the actin cytoskeleton with different signals (17-19). While some pathogenic bacteria fall prey to macrophages, others escape the immune defense program therein through either self-extrusion or creation of a protective intracellular niche $(20,21)$. Several other bacteria, mostly nonpathogenic commensals are also able to reside in macrophages without being killed $(22,23)$. Thus bacterial infections are inherently associated with the cytoskeletal actin dynamics of macrophages (24-26). However, despite considerable research and extensive knowledge in this field our understanding of how host defense mechanisms influence the cytoskeletal actin organization to regulate infection outcome remains incomplete.

Several labs including ours' have demonstrated that Wnt5A signaling induces alterations in actin assembly in macrophages $(16,24,27,28)$. This finding is in perfect agreement with the depicted role of Wnt5A in bacterial phagocytosis (15). Wnt5A induced alterations in actin assembly are in fact linked with a Rac1-Disheveled dependent host autophagy circuit that promotes both internalization and killing of pathogenic bacteria such as Pseudomonas sp., which are associated with respiratory disorders (16). Wnt5A mediated killing of Mycobacterium sp. through the host autophagy machinery has also been demonstrated (29). Interestingly, however, nonpathogenic E. coli are internalized by Wnt5A signaling, but not killed (15), this being in line with the reported survival of nonpathogenic $E$. coli in macrophages through extended time periods $(30,31)$. These differences in infection outcome led us to investigate how Wnt5A aided actin assembly controls different bacterial infections at the molecular level.

In the current report we demonstrated using pathogenic E. coli K1 and non-pathogenic E. coli K12-MG1655 and E. coli DH5 $\alpha$ that the outcome of Wnt5A assisted actin organization is different for the pathogenic and non-pathogenic strains of $E$. coli. While activation of actin assembly by Wnt5A facilitated the killing of only the pathogenic but not the non-pathogenic E. coli, intercepting activation of the Wnt5A-Actin axis by actin assembly inhibitors reversed the scenario, leading to elimination of the non-pathogen but not the pathogen. Overall, our data indicate that Wnt5A signaling controls the outcome of different bacterial infections at least partly through actin organization.

\section{MATERIALS AND METHODS}

\section{Cell Culture and Reagents}

RAW 264.7 macrophages (ATCC ${ }^{\circledR} \mathrm{TIB}^{\mathrm{TM}}{ }^{\mathrm{TM}}$ ), and mouse peritoneal macrophages were maintained under normal tissue culture conditions following published protocol (32). E. coli K1 (gift from Dr. Victor Nizet, UCSD, CA), Pseudomonas aeruginosa strain PA14 (gift from Dr. Chitra Mandal, IICB, Kolkata), E. coli DH5 $\alpha$ and E. coli K12-MG1655 (MTCC. 1586) were used to infect RAW 264.7 and peritoneal macrophages. PIPES (P1851), EGTA (E3889), Glycerol (G5516), ATP (A2383), $\mathrm{NaCl}$ (S5886), NP-40 (492018), Triton X-100 (11332481001), and Anti-Mouse HRP (A4416) were purchased from SigmaAldrich (St. Louis, MO, USA). RNAiMax Transfection Reagent (13778150) was purchased from Invitrogen (Thermo Fisher Scientific, Waltham, MA, USA). Phalloidin (A34055) and DAPI (D1306) were purchased from Molecular Probes (Eugene, OR, USA). rWnt5A (GF146), Rac1 inhibitor (NSC23766), Arp-2/3 complex inhibitor I (CK-666) \& II (CK869), Tris base (648310), and $\mathrm{Na}_{3} \mathrm{VO}_{4}$ (D00152519) were purchased from Calbiochem (San Diego, CA, USA). $\mathrm{MgCl}_{2}$ (60583305001046), Tween 20 (655205), $\beta$-mercaptoethanol (8057400250), NaF (61773705001730), PVDF membrane (IPVH00010) and Luminataclassico chemiluminescent substrate (WBLUC0500) were purchased from Millipore (Burlington, MA, USA). Anti-Wnt5A (MAB645) and Anti- Rat HRP (HAF005) antibodies were purchased from R\&D (Minneapolis, MN, USA). Anti-Actin antibody (ACTN05-C4) was purchased from Thermo Fisher Scientific (Waltham, MA, USA). On-target plus SMART Pool siRNA against murine Wnt5A (L-065884-01) and nontargeting pool control siRNA (D-001810-05) were purchased from Dharmacon (Lafayette, CO, USA). Anti- $\beta$-actin (SC-47778), Rac1 (SC-217), and Rab7 (SC376362) antibodies were purchased from Santa Cruz Biotechnologies (Dallas, TX, USA). Anti-Arp2 (Thr-237/Thr238), phospho-specific (AP3871), and Anti-Arp2 (AP3861) antibodies were purchased from ECM Biosciences (Versailles, KY, USA). DMSO (196055) was purchased from MPBiomedicals (Solon, OH, USA). cDNA synthesis kit and Taq Polymerase were purchased from BioBharati Life Sciences (Kolkata, India). RNA IsoPlus was purchased from Takara (Kusatsu, Shiga, Japan). Mouse Wnt5a: 5'-CAGGTCAACAGC CGCTTCAAC-3' (forward) 5'-ACAATCTCCGTGCACTT CTTGC-3' (reverse) and GAPDH: 5'-ACCACAGTCCATG CCATCAC-3' (forward); 5'-TCCACCACCCTGTTGCTGTA$3^{\prime}$ (reverse) primers was purchased from Integrated DNA Technologies, USA were used to conduct RT-PCR.

\section{Bacterial Killing Assay}

Cells grown to about $60 \%$ confluency were infected separately with different bacteria at MOI: 10 for $1 \mathrm{~h}$ (T0), after which the extracellular bacteria were discarded by extensive washing. Infected cells were incubated from 1 to $4 \mathrm{~h}$ (T1-T4) under normal tissue culture conditions, harvested, lysed in autoclaved distilled water and plated on agar plates for CFU (Colony Forming Units) enumeration.

For the inhibitor assay cells were infected with different bacteria for $1 \mathrm{~h}$ and post-infection different inhibitors were added to the media after PBS washes and kept for $3 \mathrm{~h}$. CFU enumeration was done both at $1 \mathrm{~h}$ (initial) and $3 \mathrm{~h}$ (final) time points. Percentage of bacteria killed was calculated by the equation: [(Initial CFU - Final CFU)/Initial CFU] $\times 100$. Calculations were controlled to cell number. 


\section{Western Blotting}

Harvested cell pellet was lysed using cell lysis buffer for $15 \mathrm{~min}$ at $4^{\circ} \mathrm{C}$ (16). Following centrifugation at $12,000 \mathrm{rpm}$ for $5 \mathrm{~min}$ at $4^{\circ} \mathrm{C}$, about $40 \mu \mathrm{g}$ of the clear lysate was run on SDS-PAGE and transferred to PVDF membrane. After blocking with 5\% BSA for $2 \mathrm{~h}$ the membrane was incubated with primary antibody at $4^{\circ} \mathrm{C}$, following which appropriate HRP-secondary antibody was added and incubation continued at room temperature. Finally, the membrane was visualized by Chemi documentation system of Azure Biosystems, Model-C400. GelQuant.Net was used for calculation of band intensities.

\section{Transfection}

Wnt5A siRNA transfection was done as reported previously (32). Briefly, RAW264.7 macrophages were plated in six-well tissue culture plates $\left(\sim 2 \times 10^{6}\right.$ cells per well $)$ a day before transfection and incubated at $37^{\circ} \mathrm{C}$ in $5 \% \mathrm{CO}_{2}$. On day of transfection, initially $0.7 \mathrm{ml}$ of medium containing $2 \%$ FBS was added to the cells. Subsequently $25 \mathrm{nM}$ Wnt5A siRNA or random siRNA was complexed with $5 \mu \mathrm{l}$ of Lipofectamine RNAiMax transfection reagent in $300 \mu$ of antibiotic-free serum-free culture medium and incubated for $30 \mathrm{~min}$ before adding to the cells. The cells were incubated for $24 \mathrm{~h}$ after which the culture medium was replaced with complete medium containing antibiotic and incubated for about $32 \mathrm{~h}$. Transfected cells were infected with bacteria for $1 \mathrm{~h}$ (T0), following which, the infection was removed and cells were kept for $3 \mathrm{~h}$ (T3) under normal tissue culture condition without antibiotic. CFU was plotted controlled to cell number.

\section{Filamentous (F) Actin Preparation and Immunoblotting}

F-actin isolation was done following published protocol (33). Briefly, cells were harvested, resuspended in F-actin Stabilization Buffer (FSB) (33) and kept at $37^{\circ} \mathrm{C}$ for 10 min following which the mix was centrifuged at 2,000 rpm to separate the debris and unbroken cells. The supernatant was centrifuged at $150,000 \mathrm{~g}$ for $60 \mathrm{~min}$ in SW61 rotor to obtain the F-actin pellet, which was resuspended in F-actin destabilizing solution $(10 \mu \mathrm{M}$ Cytochalasin D in sterile distilled water). F-actin level was estimated by immunoblotting with actin antibody.

\section{Phagosome Isolation}

Phagosome was isolated following published protocol (16). RAW264.7 cells pretreated with Wnt5A conditioned medium (L5A) and control medium (L) from L cells (32) for $6 \mathrm{~h}$ were infected with bacteria (MOI: 10) for $1 \mathrm{~h}$ without added antibiotic. After removal of bacteria and addition of fresh DMEM, incubation of infected cells was continued for 2 additional $h$ following which the harvested cells were washed with ice cold PBS (twice), resuspended in homogenization buffer [HB; $20 \mathrm{mM}$ HEPES/KOH ( $\mathrm{pH} 7.2$ ), $0.5 \mathrm{mM}$ EGTA, and $250 \mathrm{mM}$ sucrose] and left on ice for $5 \mathrm{~min}$. Following centrifugation at 2,000 rpm the cell pellet was resuspended in $2 \mathrm{ml} \mathrm{HB}$ (without EGTA) and lysed in a dounce homogenizer. After another centrifugation at $440 \mathrm{~g}$ for $3 \mathrm{~min}$ at $4^{\circ} \mathrm{C}, 2.4 \mathrm{ml}$ of $65 \%$ sucrose was added to the 2 $\mathrm{ml}$ clear homogenate to obtain 39\% final sucrose concentration. This $4.4 \mathrm{ml}$ sucrose containing cell homogenate was layered over

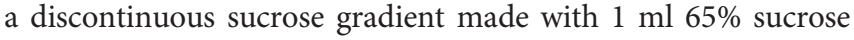
and $2 \mathrm{ml} \mathrm{55 \%}$ sucrose. Subsequently, $2 \mathrm{ml}$ of $32.5 \%$ sucrose and 1 $\mathrm{ml}$ of $10 \%$ sucrose were added on top of the cell homogenate layer. This was followed by ultracentrifugation in SW41 rotor at $100000 \mathrm{X} g$ for $1 \mathrm{~h}$. Phagosome was collected from the interface of $55 \%$ and $65 \%$ sucrose layers, pelleted by centrifugation at $18,000 \mathrm{X} g$ for $10 \mathrm{~min}$ and further resuspended in $\mathrm{HB}$. The resuspended phagosome pellet was boiled at $95^{\circ} \mathrm{C}$ for $10 \mathrm{~min}$ in SDS-PAGE 4X sample buffer for immunoblotting. During inhibitor experiment, the inhibitor was added to cells after $1 \mathrm{~h}$ of infection with the bacteria. Following $3 \mathrm{~h}$ incubation after removal of the bacteria, phagosome was prepared. For phagosome CFU calculation, phagosome pellet was resuspended in $\mathrm{HB}$ and $3 \mu \mathrm{l}$ of the suspension was added to 50 $\mu \mathrm{l}$ of autoclaved distilled water. 1:20 dilution in fresh autoclaved distilled water was plated on LB Agar plate. For western blotting, sample preparation was done by addition of $4 \mathrm{X}$ sample buffer to equal volumes of resuspended phagosome, followed by boiling at $95^{\circ} \mathrm{C}$ for $15 \mathrm{~min}$.

\section{Confocal Microscopy}

Confocal microscopy was done following previously published protocol (16). Briefly peritoneal macrophages and RAW264.7 cells were plated onto three chambered glass slides. Fixed cells (fixation was done in $4 \%$ paraformaldehyde for $15 \mathrm{~min}$ ) were stained with Phalloidin (Alexa Fluor 455, 1:2,000) and DAPI $(1: 4,000)$ in $2.5 \%$ BSA dissolved in PBST $(0.1 \%$ Tween-20) for 15 min, which was followed by $3 \times$ PBST washes. The slides were mounted and visualized under Olympus Fluoview FV10i at 60x objective and $1.6 \times$ zoom. Fluorescence intensity was measured by ImageJ.

\section{Statistical Analysis}

Results were analyzed with unpaired Student $t$ test using GraphPad Prism 6 software. Line diagrams and bar graphs are expressed as mean \pm SEM. $p \leq 0.05$ is considered statistically significant. Significance is annotated as follows: ${ }^{\star} p \leq 0.05,{ }^{* *} p \leq$ $0.005,{ }^{* *} p \leq 0.0005$.

\section{Ethics Statement}

All animal studies were approved by the Animal Ethics Committee of CSIR-IICB.

\section{RESULTS}

\section{Wnt5A Signaling Interconnects Differently With Pathogenic and Non-Pathogenic E. coli}

In order to compare the effect of Wnt5A signaling on bacterial pathogens and non-pathogens, we focused on E. coli $\mathrm{K} 1$ as a pathogen in relation to E. coli K12-MG1655 or E. coli DH5 $\alpha$ as non-pathogen. Genome comparisons reveal 19 islands, which code for different virulent factors in E. coli $\mathrm{K} 1$ but not E. coli $\mathrm{K} 12$ - 
MG1655 or E. coli DH5 $\alpha$ (34-39). Thus, to eliminate interspecies variability from our study, these bacteria were used as pathogen and non-pathogen. Wnt5A signaling was activated both in RAW 264.7 and mouse peritoneal macrophages by recombinant Wnt5A (rWnt5A) treatment $(50 \mathrm{ng} / \mathrm{ml})$ for $6 \mathrm{~h}$ prior to bacterial infection $(15,16,27)$. Subsequently, intracellular bacterial killing vs. survival was estimated by comparing the CFUs retrieved after infection for $1 \mathrm{~h}$ (T0) with those retrieved at 1-h intervals (T1-T4) during incubation of the cells post infection. CFU (T0) of E. coli K1 was always higher than that of E. coli K12-MG1655 or E. coli DH5 $\alpha$ perhaps on account of the invasiveness of this pathogenic strain. Interestingly however, in both cell types activation of Wnt5A signaling by rWnt5A increased intracellular killing of $E$. coli $\mathrm{K} 1$ as compared to the corresponding control (PBS). The internalized non-pathogenic strains K12MG1655 and DH5 $\alpha$ on the other hand, were not killed by rWnt5A (Figures 1A-F). In agreement with these findings, while E. coli K1 multiplied intracellularly upon siRNA mediated Wnt5A depletion, there was no significant multiplication of the non-pathogenic strain K12MG1655 under similar condition (Figures 1G, H). About 50\% depletion of Wnt5A mRNA and protein upon siRNA transfection is depicted in panels I, J.

\section{Interrelation Between Wnt5A Signaling and Pathogenic or Non-Pathogenic E. coli Is Associated With Actin Assembly}

The intrinsic association of cytoskeletal actin with bacterial infections $(19,40)$ led us to investigate if killing vs. survival of the pathogenic and non-pathogenic E. coli by Wnt5A signaling is associated with actin assembly.

Accordingly, we first studied the effect of the bacterial infections on actin assembly and then examined if activation of Wnt5A signaling in the infected cells introduces alterations in the scenario. Filamentous (F) actin was estimated for analyzing the extent of actin assembly. Initially, both uninfected and infected mouse peritoneal macrophages were stained with phalloidin, which binds to F-actin and visualized by confocal microscopy. As an alternative measure, F-actin of uninfected and infected macrophages was separately isolated through ultracentrifugation of suspensions of the broken cells in F-actin stabilzation buffer (33) and quantified by immunoblotting.

Confocal microscopy of phalloidin stained peritoneal macrophages after bacterial infection for $1 \mathrm{~h}$ revealed significant reduction in F-actin by E. coli $\mathrm{K} 1$, but not by $\mathrm{K} 12$ MG1655 and DH5 $\alpha$. Intensity of phalloidin stain as a measure of F-actin assembly was estimated by Image J analysis (Figure 2A, i
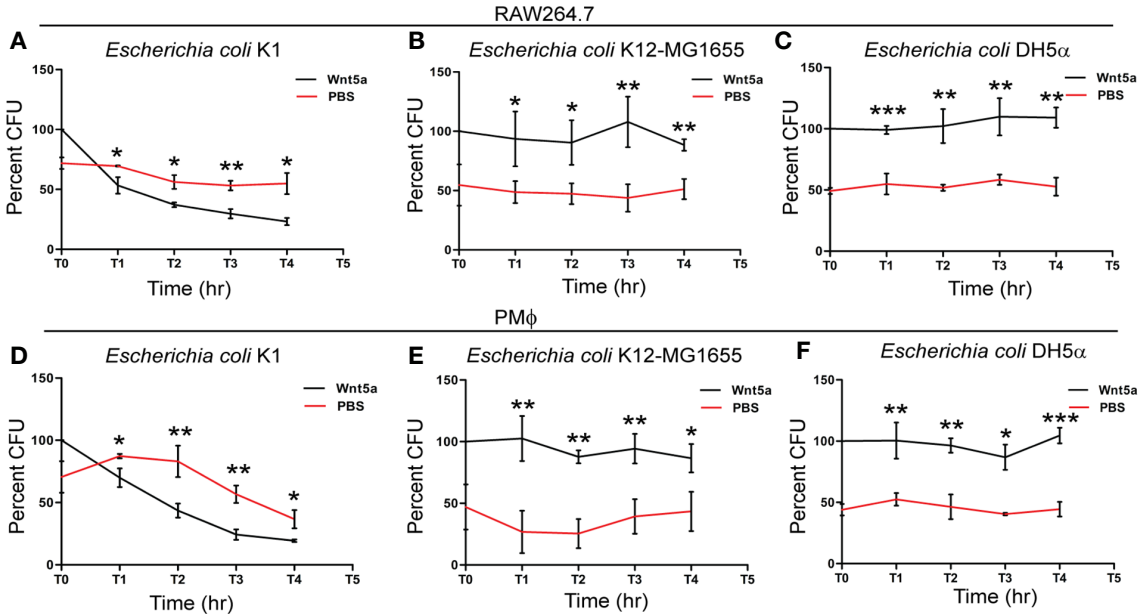

$\mathrm{PM} \phi$
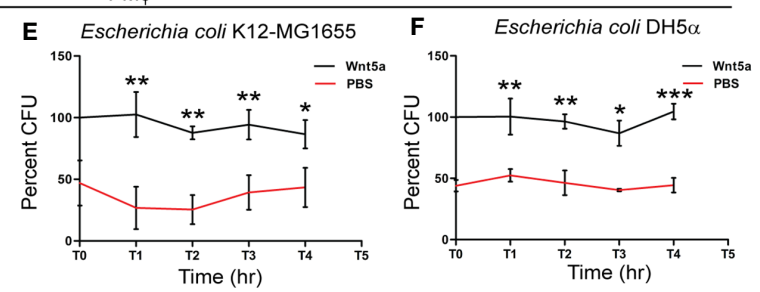

G E.coliK1 (RAW264.7)

H E.coliK12-MG1655 (RAW264.7) I

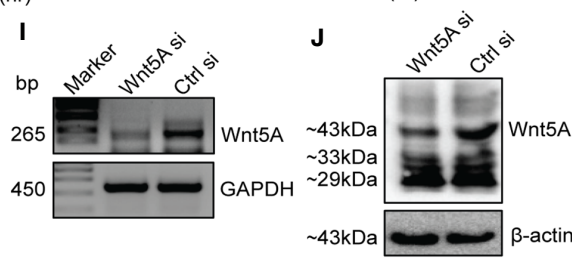

FIGURE 1 | Wnt5A signaling facilitates killing of pathogenic but not non-pathogenic E. coli. rWnt5A promoted intracellular killing of pathogenic bacterial strain $E$. coli K1 in both RAW264.7 (A) and peritoneal macrophages: PM $\phi$ (D) as estimated by Colony Forming Units (CFU) $(n=3)$ at different time points (T1-T4), $1 \mathrm{~h}$ after infection (TO) at MOI: 10, as compared to corresponding control (PBS). rWnt5A did not promote killing of non-pathogenic bacterial strains E. coli K12-MG1655 (B, E) $(n=3)$ and $E$. coli DH5 $\alpha(\mathbf{C}, \mathbf{F})(n=3)$ as observed in both RAW264.7 and PMф in comparison to corresponding control. The CFU obtained at T0 for Wnt5A treated set is considered 100 and the values in other points are normalized accordingly. Wnt5A siRNA mediated decrease in endogenous Wnt5A expression resulted in decreased uptake of both $E$. coli K1 and E. coli K12-MG1655, yet promoted intracellular proliferation of pathogenic $E$. coli $\mathrm{K} 1(\mathrm{n}=3)$ but not non-pathogenic $E$. coli K12-MG1655 $(n=3)$ as depicted by CFU at T0 and T3 (G, H). Depletion of Wnt5A expression by siRNA was confirmed by RT-PCR (I) and immunoblot analysis ( $\mathbf{( J )}$ in RAW264.7 cells. Data represented as mean $\pm \mathrm{SEM}$; ${ }^{\star} p \leq 0.05,{ }^{\star \star} p \leq 0.005,{ }^{\star \star \star} p \leq 0.0005$; NS, not significant. Wnt5Asi, Wnt5A siRNA; Crtlsi, control siRNA; Marker, DNA ladder. 

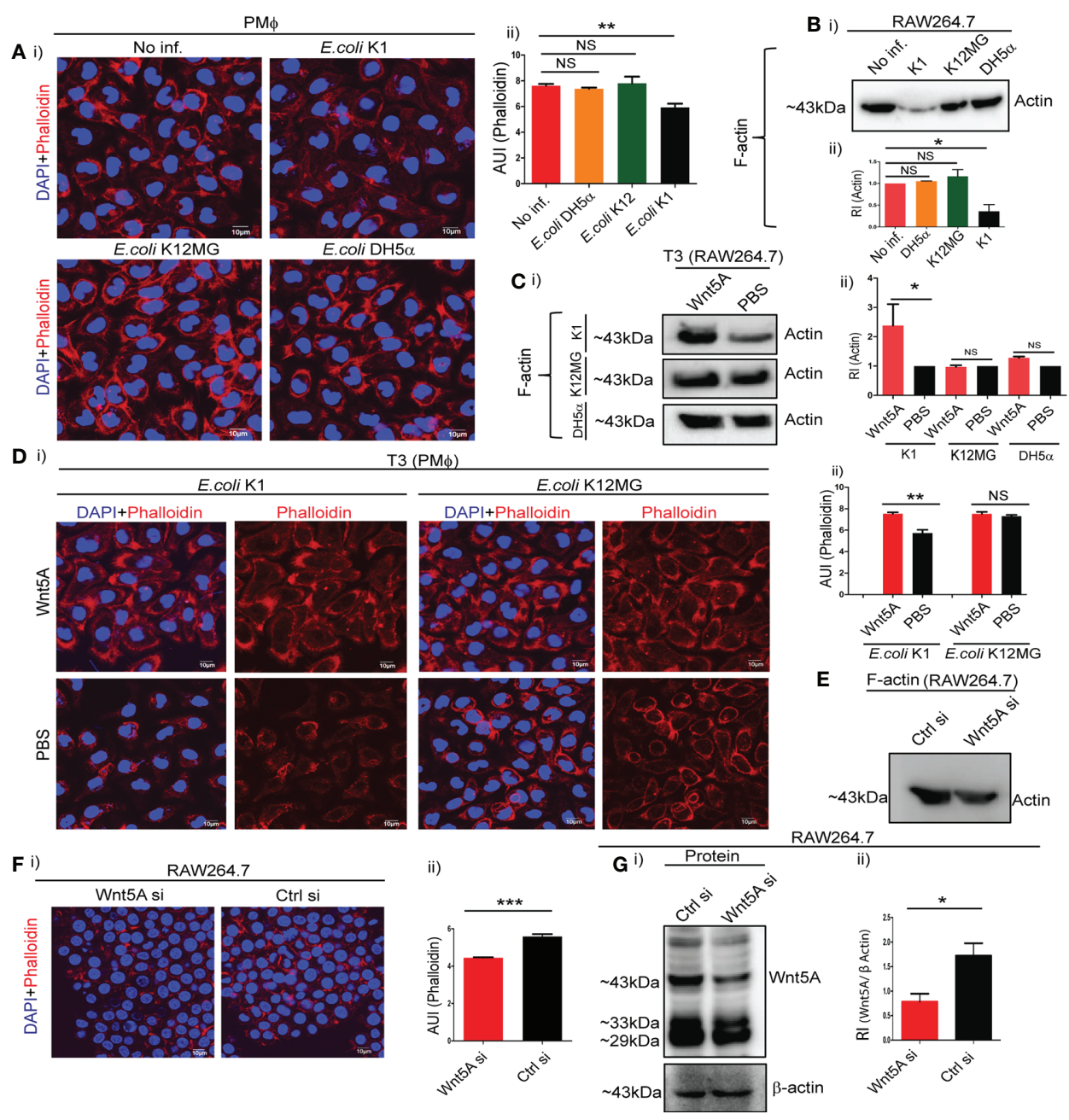

FIGURE 2 | Wnt5A signaling alters cytoskeletal actin assembly in relation to bacterial infection. Infection of PM $\phi$ and RAW264.7 by pathogenic E. coli K1, but not non-pathogenic $E$. coli K12-MG1655 or E. coli DH5 $\alpha$, at MOI:10 for $1 \mathrm{~h}$ resulted in decrease of total cellular F-actin as depicted by confocal microscopy of phalloidin stained cells (A: i, ii) and immunoblotting of isolated F-actin (B: i, ii) ( $n=3)$. Phalloidin intensity (microscopy) and actin band intensity (immunoblotting) were measured by ImageJ and GelQuant (densitometry), respectively. Wnt5A signaling opposed effect of K1 infection (3 h incubation after $1 \mathrm{~h}$ infection: T3), enhancing F-actin formation as observed by immunoblotting (C: i, ii) and confocal microscopy of phalloidin stained cells (D: $i$, ii) $(n=3)$. Wnt5A signaling produced little or no change in F actin upon E. coli K12-MG1655 and E. coli DH5 $\alpha$ infection following similar procedure (C, D). Decrease in endogenous Wnt5A level resulted in decrease of total cellular F-actin in RAW 264.7 as demonstrated by immunoblotting $(\mathbf{E})$ and confocal microscopy (F: i, ii) $(n=3)$. Depletion of Wnt5A expression by siRNA transfection was assessed through immunoblotting (G: i, ii). Data represented as mean \pm SEM; ${ }^{*} p \leq 0.05,{ }^{\star \star} p \leq 0.005,{ }^{\star \star \star} p \leq 0.0005$; NS, not significant. Phalloidin stain shown in red and DAPI (nuclear) stain shown in blue. AUl, arbitary unit of intensity; RI, relative intensity.

and ii). These findings were corroborated by immunoblotting of F-actin using RAW 264.7 macrophages. Infection of RAW264.7 cells with K1 but not K12-MG1655 and DH5 $\alpha$ led to significant reduction in the level of F-actin (Figure 2B, i and ii). Activation of Wnt5A signaling through added rWnt5A (using PBS as vehicle control), on the other hand, led to significant increase in assembled actin (F-actin) in K1 infected but not K12-MG1655 or DH5 $\alpha$ infected RAW264.7 cells during 3 h post infection (T3) (Figure 2C, i and ii), as demonstrated by immunoblotting. Phalloidin staining followed by confocal microscopy of peritoneal macrophages infected with either K1 or K12-
MG1655 after activation of Wnt5A signaling yielded the same results as projected by Image $\mathrm{J}$ analysis (Figure $2 \mathrm{D}$, $\mathrm{i}$ and ii). Thus, while significant increase in actin assembly by Wnt5 A as compared to control (PBS) in the case of E. coli $\mathrm{K} 1$ infection correlated with appreciable bacterial killing, no significant change in actin assembly by Wnt5A in the case of K12MG1655 or DH5 $\alpha$ infection correlated with survival.

That Wnt5A signaling inherently promotes actin assembly was validated by the reduced level of F-actin in Wnt5A depleted macrophages, as demonstrated by both immunoblotting and confocal microscopy (Figures 2E, F). Figure 2G demonstrates 
the siRNA-mediated reduction in Wnt5A level as compared to control. Accordingly, activation of the Wnt5A-Actin axis countered the disruptive effect of $E$. coli $\mathrm{K} 1$ on assembled actin. Since K12-MG1655 and DH5 $\alpha$ are not detrimental to assembled actin, there was no major influence of Wnt5A signaling on actin assembly in the cells infected with these strains.

\section{Different Phagosome Compositions of the Pathogen and Non-Pathogen Infected Macrophages Reflect Difference in Their Actin Organizations}

Cytoskeletal actin dynamics during bacterial internalization result in phagosome formation $(41,42)$. Since phagosomes control the fate of internalized bacteria, we examined if the respective phagosome compositions associated with pathogenic (K1) and non-pathogenic (K12-MG1655) E. coli infections in Wnt5A activated macrophages feature the observed differences in actin assembly (Figure 2). Phagosomes were harvested separately from similar numbers of E. coli $\mathrm{K} 1$ and E. coli K12MG1655 infected RAW 264.7 macrophages $3 \mathrm{~h}$ post infection and analyzed by immunoblotting (16). Prior to infection, the macrophages were activated with either Wnt5A conditioned medium prepared from Wnt5A overexpressing L cells (L5A) or treated with $\mathrm{L}$ cell conditioned medium (L) as control. Wnt5A conditioned medium, as a source of $\operatorname{Wnt5A}(15,16,32)$, was used to limit the use of the expensive rWnt5A protein.

Indeed, in case of $E$. coli $\mathrm{K} 1$ infection, phagosomes of L5A treated cells assembled significantly more actin than those of the $\mathrm{L}$ treated cells, substantiating increased Wnt5A assisted actin assembly in $\mathrm{K} 1$ infection. L5A induced increase in phagosomal actin correlated with augmented accumulation of phosphorylated Arp2 (p-Arp2: phosphorylated at Thr 237/238, Tyr.202), which is crucial for initiation of actin polymerization (43), but not unphosphorylated Arp2. Another regulator of actin assembly, Rac1 (44,45), also accumulated more in the L5A phagosomes than the L phagosomes of the K1 set. L5A mediated increased phagosomal actin assembly also correlated with phagosomal maturation as depicted by increase in Rab7, a marker of phagolysosomes (Figure 3A, lanes 1,2). This result was in accordance with increased $\mathrm{K} 1$ killing, i.e. lesser number of $\mathrm{K} 1$ in the L5A phagosomes as compared to the L phagosomes (Figure 3B, i). In case of E. coli K12-MG1655 infection, no difference in actin, Rac1, p-Arp2 or Arp2 between the L5A and L phagosomes was noted, supporting lack of significant alteration of actin assembly (Figure 3A, lanes 3, 4). In addition, there was also no increase in phagosomal maturation and activity upon stimulation with L5A, as evident from the similar number of retrieved K12-MG1655 from the L5A and L phagosomes (Figures 3A, B, i). Panels Bii, Biii \& Biv depict the L5A induced increase in phagosomal p-Arp2, actin and Rac1 with reference to unphosphorylated Arp2 in the K1 but not K12MG1655 infected macrophages, validating the occurrence of altered actin dynamics during Wnt5A assisted bacterial killing. Panel C denotes presence of Wnt5A in L5A conditioned medium but not in $\mathrm{L}$.

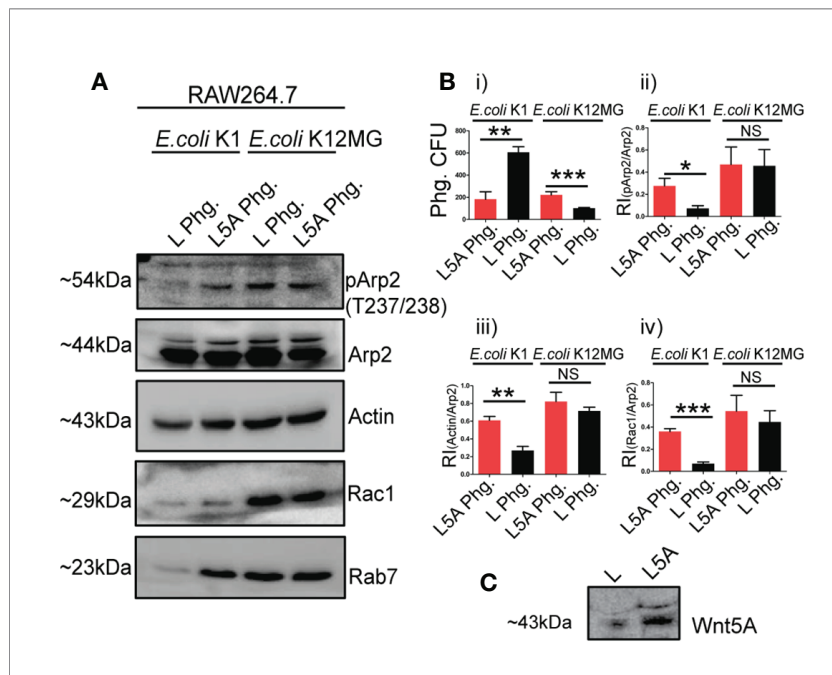

FIGURE 3 | Wnt5A (L5A) signaling aided actin assembly during pathogen vs. non-pathogen infection relates to altered phagosomal composition. Levels of phosphorylated Arp2 (pArp2), Actin, Rac1 and Rab7 with reference to unphosphorylated Arp2 was higher in phagosome of L5A treated E. coli K1 infected RAW264.7 macrophages (L5A Phg) $3 \mathrm{~h}$ post infection in comparison with the control phagosome from $L$ treated infected RAW264.7 ( $L$ Phg.) as demonstrated by immunoblotting (A: lanes 1, 2) $(\mathrm{n}=3)$. In E. coli K12MG1655 infection there was no such difference in the level of pArp2, Actin, Rac1, Rab7 between L5A Phg. and L Phg. (A: lanes 3, 4). CFU enumeration demonstrated L5A mediated killing of K1 but not K12-MG1655 at phagosome level (B.i) $(n=3)$. Bii, Biii and Biv depict the Relative Intensity (RI) of pArp2, Actin and Rac1 in relation to unphosphorylated Arp2 in L5A Phg. and L Phg of the E. coli K1 and E. coli K12-MG1655 infected sets. (C) depicts the presence of Wnt5A in L5A conditioned media (L-cells stably expressing $W n t 5 A$ ) but not in $L$ conditioned media by immunoblotting. Data represented as mean \pm SEM; ${ }^{*} p \leq 0.05,{ }^{* *} p \leq 0.005,{ }^{\star * *} p \leq 0.0005$; NS, not significant.

\section{Inhibitors of Actin Assembly Alter the Fate of $E$. coli K1 and E. coli K12MG1655/E. coli DH5 $\alpha$ Infections}

To separately validate that Wnt5A aided actin assembly is intrinsically associated with the outcome of infections with pathogenic and non-pathogenic E. coli we examined if the effect of these bacterial infections can be altered through the application of actin assembly inhibitors, which block activation of Arp2/3 complex and Rac1, thereby inhibiting actin nucleation and branching $(46,47)$.

Wnt5A or PBS (vehicle control) pretreated RAW 264.7 and mouse peritoneal macrophages were infected with either $E$. coli K1 (pathogenic) or K12-MG1655 and DH5 $\alpha$ (non-pathogenic) for $1 \mathrm{~h}$, washed free of extracellular bacteria and incubated for $3 \mathrm{~h}$ with inhibitors to Arp2/3 complex $(20 \mu \mathrm{M})$ and $\operatorname{Rac} 1(15 \mu \mathrm{M})$ using DMSO and PBS as vehicle controls, respectively $(46,47)$. Bacterial CFU retrieved from the infected cells before and after 3 $h$ incubation depicted the effect of inhibition of Wnt5A assisted actin assembly on infection outcome. Confocal microscopy of the phallodin stained cells was performed to validate inhibitor induced alteration in actin assembly.

Interestingly, Arp2/3 complex (CK666 \& CK869) and Rac1 inhibitors blocked killing of E. coli K1 but promoted 
killing of both K12-MG1655 and DH5 $\alpha$ in Wnt5A activated RAW 264.7 and mouse peritoneal macrophages (Figures 4AD and Supplementary Figures 1A, B). No notable effect of the inhibitors on the infection load in PBS (control for Wnt5A) treated macrophages suggested that the inhibitors were active only when actin assembly was stimulated by Wnt5A signaling (Supplementary Figures 2A, B). From confocal microscopy of phalloidin stained cells it was evident that the inhibitors significantly reduced actin assembly (phalloidin stain) in the Wnt5A activated $\mathrm{K} 1$ and K12-MG1655 infected macrophages. However, while in case of $\mathrm{K} 1$, the inhibition resulted in a rather low level of actin assembly, similar to the control (PBS set), in case of K12MG1655 considerable actin assembly persisted even after the inhibition (Figures 4E, F). As before, there was no significant effect on phalloidin stain in absence of stimulation by Wnt5A (Supplementary Figures 2C, D).

Differences in actin assembly in the inhibitor treated MG1655 and $\mathrm{K} 1$ infected macrophages were indeed reflected also at the phagosome level, Rab7 indicating phagosome maturation. However, although the inhibitors diminished actin assembly, there was relatively more actin, $\mathrm{p}$-Arp 2 and Rac1 in relation to
A

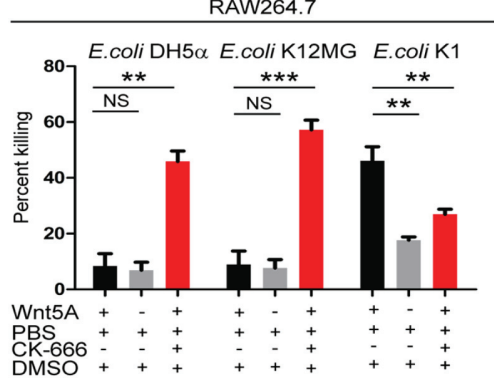

C

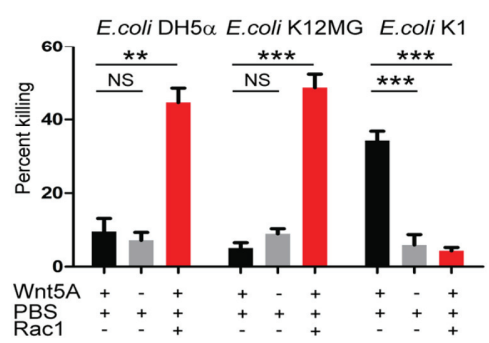

E

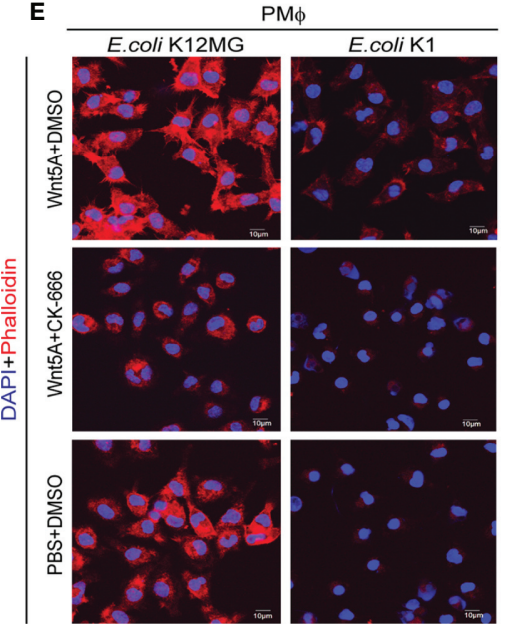

B

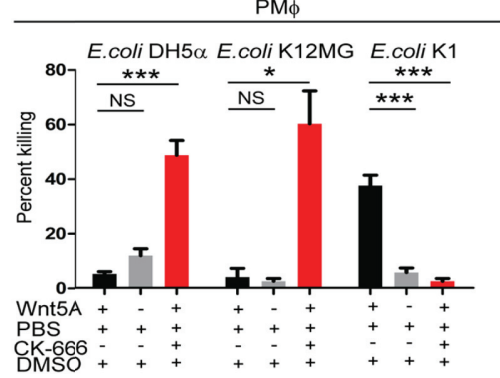

D

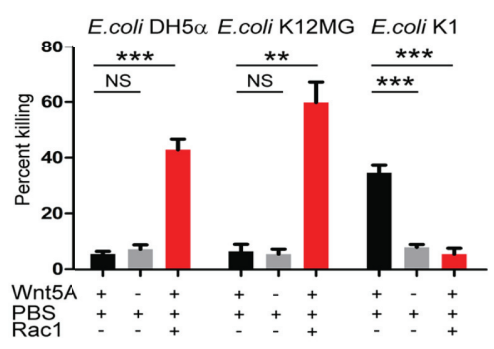

$\mathbf{F}$

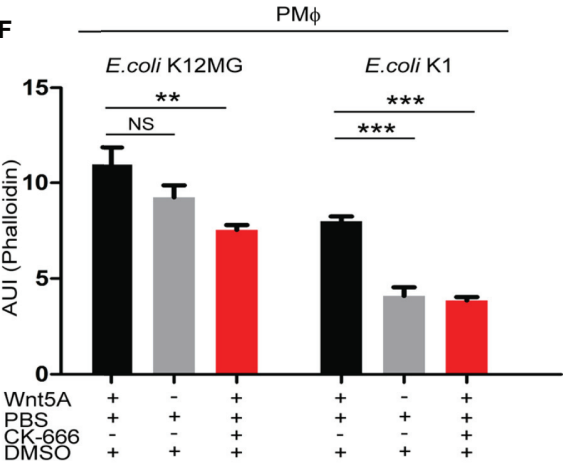

FIGURE 4 | Arp2/3 complex and Rac1 inhibitors modify the Wnt5A induced fate of pathogenic and non-pathogenic bacteria by inducing cytoskeletal actin assembly in infected macrophages. Class I Arp2/3 complex inhibitor (CK-666; $20 \mu \mathrm{M})(\mathrm{n}=3)$ and Rac1 inhibitor (Rac1; $15 \mu \mathrm{M})(\mathrm{n}=3)$ treatment post infection promoted killing of $E$. coli $\mathrm{K} 12 \mathrm{MG} 1655$ (E. coli $\mathrm{K} 12 \mathrm{MG}$ ) and $E$. coli $\mathrm{DH} 5 \alpha$ in Wnt5A activated cells but impaired the killing of $E$. coli $\mathrm{K} 1$ in both RAW 264.7 and peritoneal macrophages as presented in (A-D). Arp2/3 complex Class I inhibitor (CK-666; $20 \mu \mathrm{M}$ ) altered Wnt5A induced actin modulation both in case of E. coli K12MG1655 and $E$. coli $\mathrm{K} 1$ as detected by phalloidin staining in peritoneal macrophages and confocal microscopy. $\mathrm{F}$ actin organization of Wnt5A+CK-666 added $E$. coli $\mathrm{K} 12 \mathrm{MG}$ set was more than that of the Wnt5A+CK-666 added $E$. coli $\mathrm{K} 1$ set $(\mathbf{E}, \mathbf{F})(\mathrm{n}=4)$. DMSO+PBS was used as vehicle control for the experiment. Data represented as mean \pm SEM; ${ }^{*} p \leq 0.05,{ }^{* *} p \leq 0.005,{ }^{* * *} p \leq 0.0005$; NS, not significant. 
unphosphorylated Arp2 in the MG1655 phagosomes as compared to the K1 phagosomes (Supplementary Figure 3).

Unlike K1 infection, which antagonizes actin assembly, K12MG1655 infection does not have appreciable influence on actin assembly (Figure 2). Accordingly, the residual assembled actin after administration of actin assembly inhibitors was always notably more in K12-MG1655 infected macrophages as compared to $\mathrm{K} 1$ infected macrophages.

These results clearly indicate that optimal conditions with regard to actin assembly are required for the killing of pathogens and non-pathogens. In case of $\mathrm{K} 1$ infection, the optimal condition was obtained through activation of Wnt5A signaling, but in case of K12-MG1655 or DH5 $\alpha$ infection, additional influence of actin assembly inhibitor was required.

\section{DISCUSSION}

Host-pathogen interactions focusing on how various bacterial pathogen specific virulence factors hijack the actin cytoskeleton have been extensively described $(19,25)$. But how the host defense system incorporates the actin network to counter bacterial infections remains unclear. How bacterial nonpathogens as compared to pathogens fit in this scenario is also not clearly understood.

In view of the role of Wnt5A signaling in actin assembly (16, $24,27,28)$, we studied how the Wnt5A-Actin axis regulates the outcome of infection by a bacterial pathogen (E. coli $\mathrm{K} 1$ isolate from a biliary sepsis patient) as compared to a non-pathogen (lab strain E. coli DH5 $\alpha$ or E. coli K12-MG1655). The basis of pathogenicity and non-pathogenicity of these E. coli strains have already been documented (36-39). Thus we did not focus on how particular virulence factors affect the actin cytoskeleton during infection. Rather, we studied the influence of the Wnt5A-Actin axis on the pathogen and the non-pathogen, and vice versa.

We observed that the pathogen antagonizes actin assembly in macrophages and disrupts it, as depicted by biochemical estimation of cellular F-actin and confocal microscopy of phalloidin stained cells. But activation of Wnt5A mediated actin organization in the pathogen-infected macrophages, as ascertained by similar methodologies including analysis of phagosome composition, promotes killing of the pathogen (Figures 1, 2, and 3). Application of actin assembly inhibitors, moreover, blocks the killing promoted by Wnt5A signaling (Figure 4 and Supplementary Figure 1). On the contrary, the non-pathogens, which have no significant effect on Wnt5A mediated actin organization, remain protected by Wnt5A (Figures 1, 2, and 3). Consequently, diminution in Wnt5A assisted actin organization by actin assembly inhibitors promotes killing of the non-pathogens (Figure 4 and Supplementary Figure 1). Thus, as explained in a simple model (Supplementary Figure 4), it is the extent and type of actin organization in relation to the incumbent bacteria, which decides whether the bacteria will be killed, Wnt5A signaling being a significant player in this interplay. E. coli K12MG1655 is protected by host Wnt5A signaling because this nonpathogenic strain is compatible with Wnt5A induced actin alteration. Conversely Wnt5A signaling opposes infection by $E$. coli $\mathrm{K} 1$ facilitating its clearance because $\mathrm{K} 1$ is incompatible with Wnt5A signaling and decreases F-actin assembly. Thus, it may be stated that bacterial infections in macrophages can be managed through changes in the degree of actin assembly by regulation of Wnt5A signaling through activation by rWnt5A and application of specific inhibitors at appropriate dosages. Accordingly, Wnt5A signaling may be envisaged as a regulator of immune resistance to harmful infections. This concept is corroborated by the regulatory effect of Wnt5A signaling on infections by pathogenic bacteria such as Streptococcus pneumoniae, Pseudomonas aeruginosa, and Mycobacterium tuberculosis $(16,29)$. Incidentally, like K1 infection, infection by Pseudomonas aeruginosa (PA14) also causes decrease in polymerized actin (Supplementary Figure 5), and activation of Wnt5A signaling leads to bacterial killing (16). In connection with this study it is to be noted that Wnt5A signaling promotes the survival of the pathogen E. chaffensis (48). Hence it is important to look into the interrelation between Wnt5A assisted actin assembly and E. chaffensis infection.

Many aspects of host-mediated regulation of bacterial infections may be associated with cytoskeletal actin. These, may very well involve several TLRs, NODs, cholesterol and other lipids, and several components of the host autophagy machinery $(19,49-$ 51). For example, Pathogen Associated Molecular Patterns (PAMP) like LPS and CpG-rich bacterial DNA can facilitate actin assembly and polarity of macrophages by binding with TLR4 and TLR9, respectively (50). But, how Wnt5A signaling is involved in such TLR mediated actin dynamics is unclear. However, this is also not the focus of our study. The results summarized here clearly indicate that optimal levels and patterns of assembled actin are required for killing different bacteria, irrespective of whether these are pathogenic or non-pathogenic. Given the observed effect of Wnt5A signaling on non-pathogens, it is important to understand if commensal bacteria, which have coevolved with the host and are crucial for immune defense, benefit from Wnt5A signaling mediated actin assembly (52-54).

\section{DATA AVAILABILITY STATEMENT}

The original contributions presented in the study are included in the article/Supplementary Material. Further inquiries can be directed to the corresponding author.

\section{ETHICS STATEMENT}

The animal study was reviewed and approved by CSIR-IICBAnimal Ethics Committee.

\section{AUTHOR CONTRIBUTIONS}

MS designed research, analyzed data, and wrote the paper. SJ performed research, intellectually contributed to research design, 
analyzed data, and assisted in writing the paper. SS performed some research and assisted in paper writing. All authors contributed to the article and approved the submitted version.

\section{FUNDING}

This work was supported by a grant (BT/PR27125/BRB/10/1635/ 2017) from the Department of Biotechnology, Government of India and institutional funding. SJ was supported by Research Scholar fellowship from CSIR, Government of India and by The Company of Biologist, Journal of Cell Biology. SS was supported by Research Scholar fellowship from CSIR, Government of India.

\section{REFERENCES}

1. Nusse R, Nusse R. Wnt signaling in disease and in development. Cell Res (2005) 15:28-32. doi: 10.1038/sj.cr.7290260

2. He X, Saint-Jeannet J-P, Wang Y, Nathans J, Dawid I, Varmus H. A Member of the Frizzled Protein Family Mediating Axis Induction by Wnt-5A. Science (1997) 275:1652-4. doi: 10.1126/science.275.5306.1652

3. Cadigan KM, Nusse R. Wnt signaling: a common theme in animal development. Genes Dev (1997) 11:3286-305. doi: 10.1101/gad.11.24.3286

4. Sato A, Yamamoto H, Sakane H, Koyama H, Kikuchi A. Wnt5a regulates distinct signalling pathways by binding to Frizzled2. EMBO J (2010) 29:41-54. doi: 10.1038/emboj.2009.322

5. Yu J, Chen L, Cui B, Widhopf GF, Shen Z, Wu R, et al. Wnt5a induces ROR1/ ROR2 heterooligomerization to enhance leukemia chemotaxis and proliferation. J Clin Invest (2015) 126:585-98. doi: 10.1172/JCI83535

6. Komiya Y, Habas R. Wnt signal transduction pathways. Organogenesis (2008) 4:68-75. doi: 10.4161/org.4.2.5851

7. Dijksterhuis JP, Petersen J, Schulte G. International Union of Basic and Clinical Pharmacology Review: WNT/Frizzled signalling: receptor-ligand selectivity with focus on FZD-G protein signalling and its physiological relevance: IUPHAR Review 3. Br J Pharmacol (2014) 171:1195-209. doi: 10.1111/bph.12364

8. Sen M, Ghosh G. Transcriptional outcome of Wnt-Frizzled signal transduction in inflammation: evolving concepts. J Immunol (2008) 181:4441-5. doi: 10.4049/jimmunol.181.7.4441

9. Clevers H. Wnt/ $\beta$-Catenin Signaling in Development and Disease. Cell (2006) 127:469-80. doi: 10.1016/j.cell.2006.10.018

10. Kühl M, Sheldahl LC, Park M, Miller JR, Moon RT. The Wnt/Ca2+ pathway: a new vertebrate Wnt signaling pathway takes shape. Trends Genet (2000) 16:279-83. doi: 10.1016/S0168-9525(00)02028-X

11. Chavali M, Klingener M, Kokkosis AG, Garkun Y, Felong S, Maffei A, et al. Non-canonical Wnt signaling regulates neural stem cell quiescence during homeostasis and after demyelination. Nat Commun (2018) 9:1-17. doi: 10.1038/s41467-017-02440-0

12. Witze ES, Litman ES, Argast GM, Moon RT, Ahn NG. Wnt5a control of cell polarity and directional movement by polarized redistribution of adhesion receptors. Science (2008) 320:365-9. doi: 10.1126/science.1151250

13. Aznar N, Midde KK, Dunkel Y, Lopez-Sanchez I, Pavlova Y, Marivin A, et al. Daple is a novel non-receptor GEF required for trimeric $G$ protein activation in Wnt signaling. Elife (2015) 4:e07091. doi: 10.7554/eLife.07091

14. Mikels AJ, Nusse R. Purified Wnt5a Protein Activates or Inhibits $\beta$-CateninTCF Signaling Depending on Receptor Context. PloS Biol (2006) 4:e115. doi: 10.1371/journal.pbio.0040115

15. Maiti G, Naskar D, Sen M. The Wingless homolog Wnt5a stimulates phagocytosis but not bacterial killing. Proc Natl Acad Sci (2012) 109:166005. doi: 10.1073/pnas.1207789109

16. Jati S, Kundu S, Chakraborty A, Mahata SK, Nizet V, Sen M. Wnt5A Signaling Promotes Defense Against Bacterial Pathogens by Activating a Host Autophagy Circuit. Front Immunol (2018) 9:679. doi: 10.3389/fimmu. 2018.00679

\section{ACKNOWLEDGMENT}

We acknowledge A. Konar, IICB for animal experiments, B. Das and B Sengupta, IICB for confocal microscopy, and S.N Bhattacharya and CSIR-IICB Central Instrumentation Facility (CIF), for instrumental support.

\section{SUPPLEMENTARY MATERIAL}

The Supplementary Material for this article can be found online at: https://www.frontiersin.org/articles/10.3389/fimmu.2020.628191/ full\#supplementary-material

17. Rougerie P, Miskolci V, Cox D. Generation of membrane structures during phagocytosis and chemotaxis of macrophages: role and regulation of the actin cytoskeleton. Immunol Rev (2013) 256. doi: 10.1111/imr.12118

18. Möller J, Lühmann T, Chabria M, Hall H, Vogel V. Macrophages lift off surface-bound bacteria using a filopodium-lamellipodium hook-and-shovel mechanism. Sci Rep. Intechopen (2013) 3. doi: 10.1038/srep02884

19. Anes E. Acting on Actin During Bacterial Infection. In: Cytoskeleton Structure, Dynamics, Function and Disease. Intechopen (2017). doi: $10.5772 / 66861$

20. Thi EP, Lambertz U, Reiner NE. Sleeping with the Enemy: How Intracellular Pathogens Cope with a Macrophage Lifestyle. PloS Pathog (2012) 8:e1002551. doi: 10.1371/journal.ppat.1002551

21. Leseigneur C, Lê-Bury P, Pizarro-Cerdá J, Dussurget O. Emerging Evasion Mechanisms of Macrophage Defenses by Pathogenic Bacteria. Front Cell Infect Microbiol (2020) 10:577559. doi: 10.3389/fcimb.2020.577559

22. Morikawa M, Tsujibe S, Kiyoshima-Shibata J, Watanabe Y, Kato-Nagaoka N, Shida K, et al. Microbiota of the Small Intestine Is Selectively Engulfed by Phagocytes of the Lamina Propria and Peyer's Patches. PloS One (2016) 11: e0163607. doi: 10.1371/journal.pone.0163607

23. Rios D, Wood MB, Li J, Chassaing B, Gewirtz AT, Williams IR. Antigen sampling by intestinal $\mathrm{M}$ cells is the principal pathway initiating mucosal IgA production to commensal enteric bacteria. Mucosal Immunol (2016) 9:90716. doi: $10.1038 / \mathrm{mi} .2015 .121$

24. Jati S, Sarraf TR, Naskar D, Sen M. Wnt Signaling: Pathogen Incursion and Immune Defense. Front Immunol (2019) 10:2551. doi: 10.3389/fimmu.2019.02551

25. Mostowy S, Shenoy AR. The cytoskeleton in cell-autonomous immunity: structural determinants of host defence. Nat Rev Immunol (2015) 15:559-73. doi: $10.1038 /$ nri3877

26. Kolonko M, Geffken AC, Blumer T, Hagens K, Schaible UE, Hagedorn M. WASH-driven actin polymerization is required for efficient mycobacterial phagosome maturation arrest. Cell Microbiol (2014) 16:232-46. doi: 10.1111/ cmi.12217

27. Chakraborty A, Kurati SP, Mahata SK, Sundar S, Roy S, Sen M. Wnt5a Signaling Promotes Host Defense against Leishmania donovani Infection. J Immunol (2017) 199:992-1002. doi: 10.4049/jimmunol.1601927

28. Jati S, Sen M. Wnt Signaling Regulates Macrophage Mediated Immune Response to Pathogens. In: Macrophage Activation - Biology and Disease. Intechopen (2019). doi: 10.5772/intechopen.86433

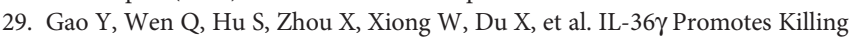
of Mycobacterium tuberculosis by Macrophages via WNT5A-Induced Noncanonical WNT Signaling. I Immunol (2019) 203:922-35. doi: 10.4049/ jimmunol.1900169

30. Häcker H, Fürmann C, Wagner H, Häcker G. Caspase-9/-3 Activation and Apoptosis Are Induced in Mouse Macrophages upon Ingestion and Digestion of Escherichia coli Bacteria. J Immunol (2002) 169:3172-9. doi: 10.4049/ jimmunol.169.6.3172

31. Goeser L, Fan T-J, Tchaptchet S, Stasulli N, Goldman WE, Sartor RB, et al. Small Heat-Shock Proteins, IbpAB, Protect Non-Pathogenic Escherichia coli from Killing by Macrophage-Derived Reactive Oxygen Species. PloS One (2015) 10:e0120249. doi: 10.1371/journal.pone.0120249 
32. Naskar D, Maiti G, Chakraborty A, Roy A, Chattopadhyay D, Sen M. Wnt5aRac1-NF- B Homeostatic Circuitry Sustains Innate Immune Functions in Macrophages. J Immunol (2014) 192:4386-97. doi: 10.4049/ jimmunol.1302817

33. Mueller P, Quintana A, Griesemer D, Hoth M, Pieters J. Disruption of the cortical actin cytoskeleton does not affect store operated Ca2+ channels in human T-cells. FEBS Lett (2007) 581:3557-62. doi: 10.1016/ j.febslet.2007.06.068

34. Sukumaran SK, Selvaraj SK, Prasadarao NV. Inhibition of apoptosis by Escherichia coli $\mathrm{K} 1$ is accompanied by increased expression of BclXL and blockade of mitochondrial cytochrome c release in macrophages. Infect Immun (2004) 72:6012-22. doi: 10.1128/IAI.72.10.6012-6022.2004

35. Blattner FR, Plunkett G, Bloch CA, Perna NT, Burland V, Riley M, et al. The complete genome sequence of Escherichia coli K-12. Science (1997) 277:145362. doi: 10.1126/science.277.5331.1453

36. Moriel DG, Bertoldi I, Spagnuolo A, Marchi S, Rosini R, Nesta B, et al. Identification of protective and broadly conserved vaccine antigens from the genome of extraintestinal pathogenic Escherichia coli. PNAS (2010) 107:9072-7. doi: 10.1073/pnas.0915077107

37. Alkeskas A, Ogrodzki P, Saad M, Masood N, Rhoma NR, Moore K, et al. The molecular characterisation of Escherichia coli $\mathrm{K} 1$ isolated from neonatal nasogastric feeding tubes. BMC Infect Dis (2015) 15:449. doi: 10.1186/ s12879-015-1210-7

38. Kuhnert P, Nicolet J, Frey J. Rapid and accurate identification of Escherichia coli K-12 strains. Appl Environ Microbiol (1995) 61:4135-9. doi: 10.1128/ AEM.61.11.4135-4139.1995

39. Grant SG, Jessee J, Bloom FR, Hanahan D. Differential plasmid rescue from transgenic mouse DNAs into Escherichia coli methylation-restriction mutants. Proc Natl Acad Sci U S A (1990) 87:4645-9. doi: 10.1073/ pnas.87.12.4645

40. Bugalhão JN, Mota LJ, Franco IS. Bacterial nucleators: actin' on actin. Pathog Dis (2015) 73. doi: 10.1093/femspd/ftv078

41. Marion S, Hoffmann E, Holzer D, Clainche CL, Martin M, Sachse M, et al. Ezrin Promotes Actin Assembly at the Phagosome Membrane and Regulates Phago-Lysosomal Fusion. Traffic (2011) 12:421-37. doi: 10.1111/j.16000854.2011.01158.x

42. Marie-Anaïs F, Mazzolini J, Herit F, Niedergang F. Dynamin-Actin Cross Talk Contributes to Phagosome Formation and Closure. Traffic (2016) 17:487-99. doi: 10.1111/tra.12386

43. Narayanan A, Iii LLL, Barber DL, Jacobson MP. Phosphorylation of the Arp2 Subunit Relieves Auto-inhibitory Interactions for Arp2/3 Complex Activation. PloS Comput Biol (2011) 7:e1002226. doi: 10.1371/ journal.pcbi.1002226

44. Sun CX, Magalhães MAO, Glogauer M. Rac1 and Rac2 differentially regulate actin free barbed end formation downstream of the fMLP receptor. J Cell Biol (2007) 179:239-45. doi: 10.1083/jcb.200705122
45. Marston DJ, Anderson KL, Swift MF, Rougie M, Page C, Hahn KM, et al. High Racl activity is functionally translated into cytosolic structures with unique nanoscale cytoskeletal architecture. PNAS (2019) 116:1267-72. doi: 10.1073/ pnas. 1808830116

46. Nolen BJ, Tomasevic N, Russell A, Pierce DW, Jia Z, McCormick CD, et al. Characterization of two classes of small molecule inhibitors of Arp2/3 complex. Nature (2009) 460:1031-4. doi: 10.1038/nature08231

47. Gao Y, Dickerson JB, Guo F, Zheng J, Zheng Y. Rational design and characterization of a Rac GTPase-specific small molecule inhibitor. Proc Natl Acad Sci U S A (2004) 101:7618-23. doi: 10.1073/pnas.0307512101

48. Luo T, Dunphy PS, Lina TT, McBride JW. Ehrlichia chaffeensis Exploits Canonical and Noncanonical Host Wnt Signaling Pathways To Stimulate Phagocytosis and Promote Intracellular Survival. Infect Immun (2016) 84:686-700. doi: 10.1128/IAI.01289-15

49. Anes E, Kühnel MP, Bos E, Moniz-Pereira J, Habermann A, Griffiths G. Selected lipids activate phagosome actin assembly and maturation resulting in killing of pathogenic mycobacteria. Nat Cell Biol (2003) 5:793-802. doi: $10.1038 /$ ncb 1036

50. Wenzel J, Held C, Palmisano R, Teufel S, David J-P, Wittenberg T, et al. Measurement of TLR-Induced Macrophage Spreading by Automated Image Analysis: Differential Role of Myd88 and MAPK in Early and Late Responses. Front Physiol (2011) 2:71. doi: 10.3389/fphys.2011.00071

51. Wickramarachchi D, Theofilopoulos AN, Kono DH. Immune Pathology Associated with Altered Actin Cytoskeleton Regulation. Autoimmunity (2010) 43:64-75. doi: 10.3109/08916930903374634

52. Molina MA, Díaz AM, Hesse C, Ginter W, Gentilini MV, Nuñez GG, et al. Immunostimulatory Effects Triggered by Enterococcus faecalis CECT7121 Probiotic Strain Involve Activation of Dendritic Cells and Interferon-Gamma Production. PloS One (2015) 10:e0127262. doi: 10.1371/journal.pone.0127262

53. Macpherson AJ, Uhr T. Induction of protective IgA by intestinal dendritic cells carrying commensal bacteria. Science (2004) 303:1662-5. doi: 10.1126/ science. 1091334

54. Ost KS, Round JL. A Few Good Commensals: Gut Microbes Use IFN- $\gamma$ to Fight Salmonella. Immunity (2017) 46:977-9. doi: 10.1016/j.immuni. 2017.06.010

Conflict of Interest: The authors declare that the research was conducted in the absence of any commercial or financial relationships that could be construed as a potential conflict of interest.

Copyright (c) 2021 Jati, Sengupta and Sen. This is an open-access article distributed under the terms of the Creative Commons Attribution License (CC BY). The use, distribution or reproduction in other forums is permitted, provided the original author(s) and the copyright owner(s) are credited and that the original publication in this journal is cited, in accordance with accepted academic practice. No use, distribution or reproduction is permitted which does not comply with these terms. 